\title{
Experiments in Aligning Threaded Parts Using a Robot
}

\author{
Hand
}

\author{
M. A. Diftler, I. D. Walker*†
}

March 5, 1998

\begin{abstract}
Techniques for determining and correcting threaded part alignment using force and angular position data are developed to augment currently limited techniques for aligning threaded parts. These new techniques are based on backspinning a nut with respect to a bolt and measuring the force change that occurs when the bolt "falls" into the nut. Kinematic models that describe the relationship between threaded parts during backspinning are introduced and are used to show how angular alignment may be determined. The models indicate how to distinguish between the aligned and misaligned cases of a bolt and a nut connection by using axial force data only. In addition, by tracking the in-plane relative attitude of the bolt during spinning, data can be obtained on the direction of the angular misalignment which, in turn, is used to correct
\end{abstract}

*M. A. Diftler is with the Automation and Robotics Department, Lockheed Martin, Houston, Texas. E-mail: mdiftler@ems.jsc.nasa.gov.

${ }^{\dagger} \mathrm{I}$. D. Walker is with the Department of Electrical and Computer Engineering, Clemson University, Clemson, South Carolina, 29634 E-mail: ianw@ces.clemson.edu. 
the misalignment. Results from experiments using a bolt held in a specialized fixture and a three fingered Stanford/JPL hand are presented.

Keywords: Threaded Fasteners, Robot Hands, Bolts, Part Mating, Part Alignment, Force Control.

\section{Introduction}

Aligning, and mating threaded parts presents significant challenges to the robotics systems designer. Bringing together a male and female part, ranging from a high tolerance bolt and nut to a low tolerance plastic jar and its cover, requires an array of robotic and artificial intelligence skills. The individual items must be identified using machine vision, haptic sensing, or perhaps a well structured world model. Both parts must be acquired and oriented properly through the use of generic grippers, robot hands, or specialized fixtures. A manipulator must perform gross alignment to bring the parts into close proximity. Either the same or another manipulator device must then finely align the parts before mating may start. The manipulators will perform both levels of alignment using either prestored or sensed information that describes relative positions. Actual part mating and tightening can only be accomplished with a combination of force and position control.

All of the above steps are essential for successful mating of threaded parts, and they can all be subdivided, yielding even more areas of on-going research. The focus of cooperative work between Clemson University, Rice University and the NASA/Johnson Space Center is the fine alignment needed to bring the parts together, and the force and position control required to successfully start thread mating, prevent wedging and jamming, and recognize 
task completion.

In a well constrained environment, such as a factory floor, alignment may not be an issue. However in less constrained and more flexible environments like on the International Space Station or on a planetary base, the position and orientation data required to align threaded parts will not always be available a priori. Experiments using the Johnson Space Center's Dexterous Anthropomorphic Robotic Testbed (DART) [6] are addressing this thread alignment issue.

The majority of the literature that deals with robotic manipulation of threaded fasteners avoids the fine alignment issue and focuses more on task decomposition [16], [8], recognizing task completion [14], [5], identifying failures [15],[14],[7], [11], and demonstrating force feedback implementations [5], [10], [13], [18], [19]. Nicolson and Fearing [9] is one of the few efforts that addresses the initial alignment issue, but even here the force sensing device used did not have the resolution necessary to identify when proper alignment was achieved.

In addition, previous systems, [9], [14], have relied on the required tightening torque to determine if the bolt and nut are in proper alignment. This technique does not provide information on how to correct alignment. A sufficiently high torque sensed at the bolt just indicates that cross threading has occurred, that the insertion has failed and, in most cases, that the parts have been damaged. A torque within specific bounds indicates that the alignment was sufficient to have successful mating between the first set of threads. In this paper, part backspinning along with axial force data and relative position data provide the basis for determining alignment and, in certain situations, information to correct alignment.

The following is an extension of previous research performed at the Johnson Space Center 
and presented in [3], [4]. Higher fidelity kinematic models are presented that show the relationship between threaded part backspinning, angular alignment, and axial force data. A specialized fixture is used to validate the models. Using both the theoretical and test data, techniques for identifying proper alignment and correcting misalignments on the order of 10 to 15 degrees are developed and tested with a Stanford/JPL hand [12].

\section{Thread Kinematics}

Backspinning a female threaded part, a nut, with respect to a male threaded part, a bolt, results in a drop of the bolt into the nut at some point during the turning process. This alignment indicator is often used by people if they are doing the task blindly. When a person feels the characteristic click of good alignment, they will then turn in the proper direction to tighten the fasteners. In this paper, the mating parts of interest are a standard steel bolt with external threads and an aluminum nut plate with a steel thread insert $\left(H E L I C O I L^{T M}\right)$. Thread inserts of this type are used in aluminum hardware for space applications. Both the bolt and the nut threads follow a helical pattern. Figure 1 shows a cross section cut of both the nut and bolt. The cross section is aligned with the thread start on the nut. Both the bolt and nut threads are modeled as equilateral triangles with the tips cut off. This is an approximation to the rounded tooth profiles that actually exist [9]. The steel insert, which is used for the nut thread, starts at full size, while the bolt is tapered on the bottom resulting in a thread that grows from zero cross section to full size after several revolutions around the bolt. 


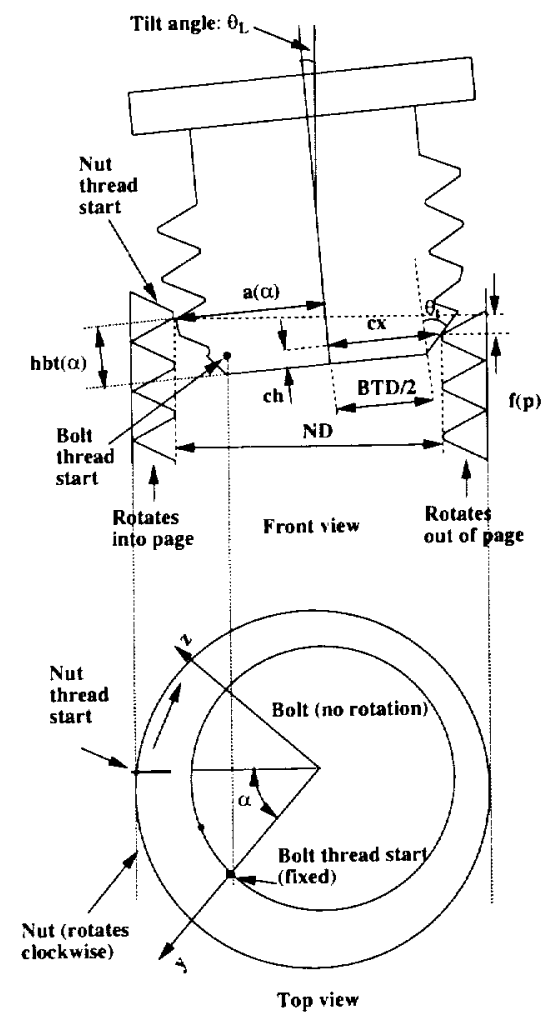

Figure 1: Bolt-nut 2 point contact

\subsection{Drop Azimuth}

The first important parameter of interest in backspinning kinematics is the point at which bolt drops into the nut during the backspinning cycle. The point at which the drop occurs will be called the "drop azimuth" and is defined by the relative angle, $\alpha$, between the thread starts of each part. Figure 1 shows the thread starts for each part from two orientations. In the experiments described in this work, the nut plate rotates while the bolt is set at various tilt angles with respect to the nut's normal. As the nut rotates, its thread start comes in contact with smaller bolt threads. When the nut reaches a cross section of the bolt that will fit within the nut's diameter, the bolt falls into the nut.

A 2 point boundary problem defines when the drop occurs and is used to calculate the 
local tilt angle, $\theta_{L}$ as a function of $\alpha$. The local tilt angle, $\theta_{L}$, is the component of the actual or global tilt between the bolt and nut that lies in the plane aligned with the nut thread start. Figure 1 illustrates the contact and the constraints are defined by:

$$
\begin{gathered}
(a(\alpha)+c x) \cos \left(\theta_{L}\right)+(h b t(\alpha)-c h) \sin \left(\theta_{L}\right)=N D \\
(h b t(\alpha)-c h) \cos \left(\theta_{L}\right)-(a(\alpha)+c x) \sin \left(\theta_{L}\right)=f(p) \\
c x=\frac{B T D}{2}+c h \tan \left(\theta_{t}\right)
\end{gathered}
$$

where $a(\alpha)$ and $h b t(\alpha)$ define the size of the bolt thread that is in contact with the nut thread start. These variables are a function of $\alpha$ since the nut comes in contact with shrinking bolt threads as it rotates. $B T D / 2$ is half the bolt diameter at the base of the bolt taper, $\theta_{t}$ is the taper angle (nominally 22.5 degrees), $N D$ is the nut diameter, and $f(p)$ is the relevant vertical distance between the nut threads in the plane and is a function of thread pitch.

The actual or global tilt angle, $\theta$, is calculated by:

$$
\theta=\theta_{L} / \cos (\alpha)
$$

The global tilt angle may also be visualized as the tilt between the nut's and bolt's vertical axes.

The importance of equation 4 and the drop azimuth angle on bolt alignment are seen in figure 2. This figure shows the actual tilt angle, $\theta$, at which a drop will occur as a function of drop azimuth, $\alpha$, in the bolt thread start, $-r_{z}$ direction (figure 1 ). The vertical line at 


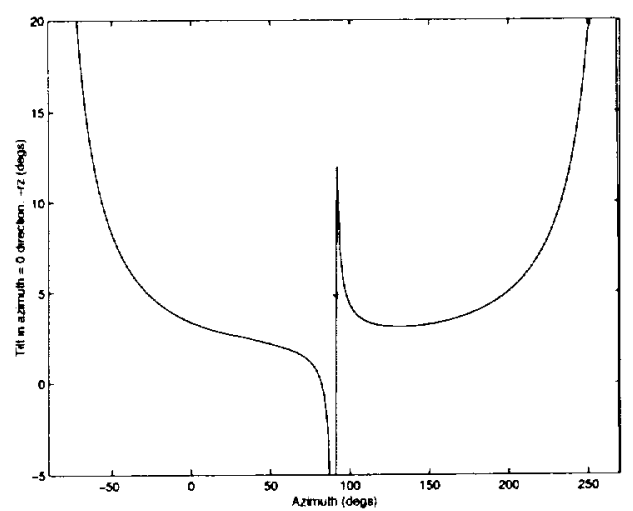

Figure 2: Drop model: tilt vs. drop azimuth $\left(-r_{z}\right.$ direction $)$

90 degrees represents a flip from positive to negative tilt angles and is a discontinuity in the model associated with cos90. (This vertical line is a graphical artifact and not part of the predicted tilt angles.) The asymptotes associated with the actual tilt angle, $\theta$, are also a result of the cosine term. Two of the asymptotes are actually the same: -90 degrees and 270 degrees. The drop azimuth is actually approaching the same asymptote from two different directions. This is an extremely important theoretical result and in simple terms states that the drop azimuth is nearly perpendicular to the tilt direction for moderate to high tilt angles. If this theoretical result correlates well with actual threaded parts and is true for all tilt directions, an initial technique to correct misalignment will be to realign the bolt by tilting in the direction perpendicular to the plane aligned with the drop azimuth $\alpha$.

A CAD representation illustrates a typical case for 10 degrees of tilt in direction of the bolt thread start. Figure 3 shows the two point contact; the cross section of the bolt gives a clearer picture of how the bolt interacts with the nut's flats. In addition, the figure shows that the global tilt, $\theta$, is for the most part into the page, yielding a drop azimuth of -58 degrees which is approaching the perpendicular $(-90)$ to the local tilt plane. 
Tilt angle $=10.62 \mathrm{degs}, \quad$ Azimuth $=\mathbf{- 5 8} \mathrm{degs}$

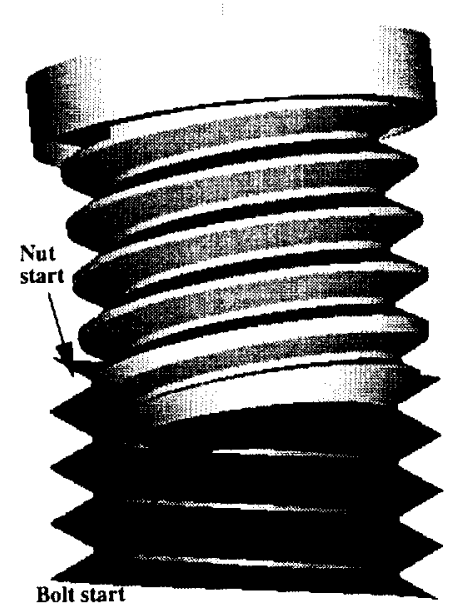

a: 2 Point contact

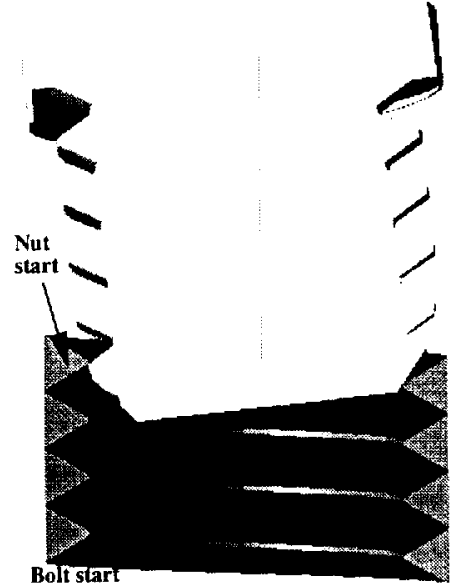

b: Bolt cross_section

Figure 3: Typical 2 point contact representation

\subsection{Drop Distance}

The second important parameter associated with backspinning is the distance the bolt drops. For this case the relevant plane of interest is the global tilt plane. This is the plane aligned with the global tilt angle. Prior to a drop the minimum distance between the next thread up on the bolt and the relevant nut thread will fall very near a point in line with the global tilt angle, $\theta[1]$.

Figure 4 presents a side view of the 10 degree tilt case from figure 3 and clearly shows that the second bolt thread is at roughly minimum distance from the nut along the plane defined by the global tilt angle. This minimum distance is the limiting factor in how far the bolt can fall into the nut.

Next, two drop models that bound the possible drop distance are constructed. Both models assume that the global tilt angle, $\theta$, does not change after the drop. The first model, the two point model, is based on a two point contact that occurs after the bolt falls. 


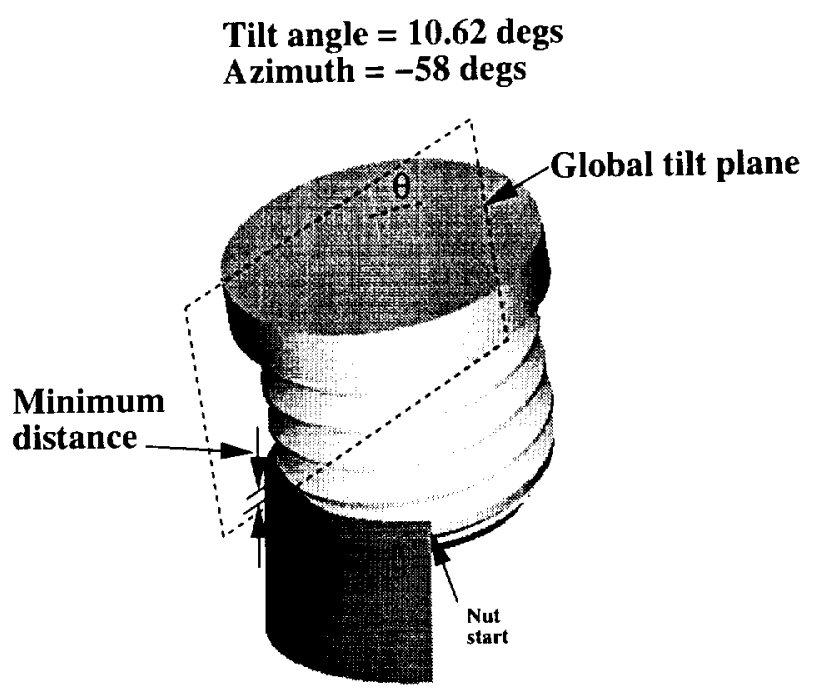

\section{Local model}

Figure 4: Side view of typical pre-drop case

Within this model, the two point contact may result in either an aligned or cross threaded configuration. In the aligned case, figure 5 , the cross section shows that the bolt is at a sufficiently small tilt angle such that both sides fall evenly, resulting in matching threads with respect to the nut. The final position after the drop is obtained from:

$$
\begin{gathered}
\left(a d\left(\alpha_{g}\right)+c x d\right) \cos \theta+\left(h d\left(\alpha_{g}\right)-c h d\right) \sin \theta=N D D \\
-\left(a d\left(\alpha_{g}\right)+c x d\right) \sin \theta+\left(h d\left(\alpha_{g}\right)-c h d\right) \cos \theta=d h d \\
N D D=N D+\frac{d h d-(f(p)+2 * D N C)}{\tan 30} \\
c x d=\frac{B D}{2}-T C+\frac{c h d-h t 1\left(\alpha_{g}-180\right)+T C H}{\tan 30}
\end{gathered}
$$

In this case the relevant bolt parameters are a function of $\alpha_{g} . \alpha_{g}$ is an angle that quantifies the global tilt plane with respect to the bolt thread start and is very similar to $\alpha$ which 


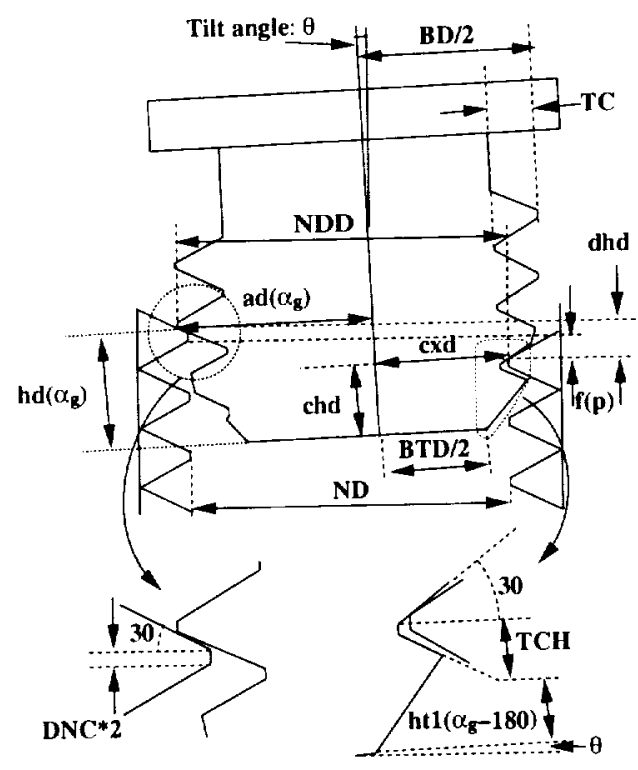

Figure 5: Two point contact drop - non-cross threading

identifies the direction of the local tilt plane. The actual drop distance is calculated by:

$$
\Delta h=f\left(h d\left(\alpha_{g}-h b t(\alpha)\right)\right.
$$

where $h b t(\alpha)$ is defined in figure 1 .

Figure 6 shows the cross threading configuration that occurs when the bolt drops unevenly and the right bolt thread does not fall. Once again, the tilt angle does not change as a result of the drop and the final position after the drop is obtained from:

$$
\begin{gathered}
\left(a d c\left(\alpha_{g}\right)+c x d c\right) \cos \theta+\left(h d c\left(\alpha_{g}\right)-c h d c\right) \sin \theta=N D D \\
-\left(a d c\left(\alpha_{g}\right)+c x d c\right) \sin \theta+\left(h d c\left(\alpha_{g}\right)-c h d c\right) \cos \theta=d h d c \\
c x d c=\frac{B T D}{2}+c h d c \tan \left(\theta_{t}\right)
\end{gathered}
$$




$$
N D D=N D+\frac{d h d c-(f(p)+2 * D N C)}{\tan 30}
$$

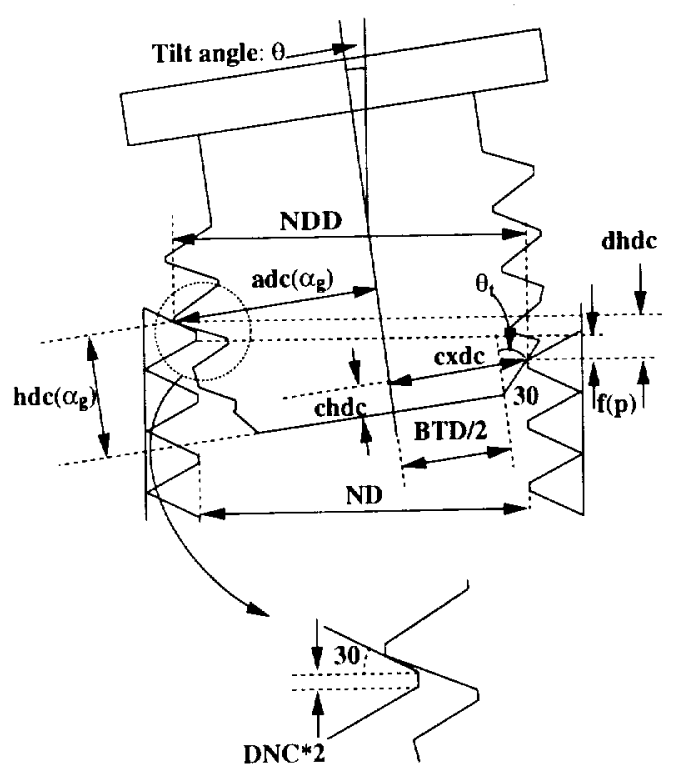

Figure 6: Two point contact drop - cross threading

The second drop model assumes a slide in the direction of the global tilt angle until contact is limited by the nut thread in the plane. This slide model is useful in describing what happens when out of plane thread contacts push the bolt in the tilt direction. This extreme case which predicts smaller drops than the two point contact drop model, is depicted in figures 7, 8, and parallels the drop model developed in [4] for the local tilt plane. The constraints are limited to one side of the bolt nut pair with two point contact between one nut tooth and the bolt. The displacement of the bolt relative to the nut is given by:

$$
\begin{gathered}
\frac{d 4}{\sin (60-\theta)}=\frac{2 * D N C}{\sin (60+\theta)} \\
\frac{d 3+d 4}{\sin 60}=\frac{\operatorname{atc}\left(\alpha_{g}\right) / \cos 30}{\sin (120-\theta)},
\end{gathered}
$$




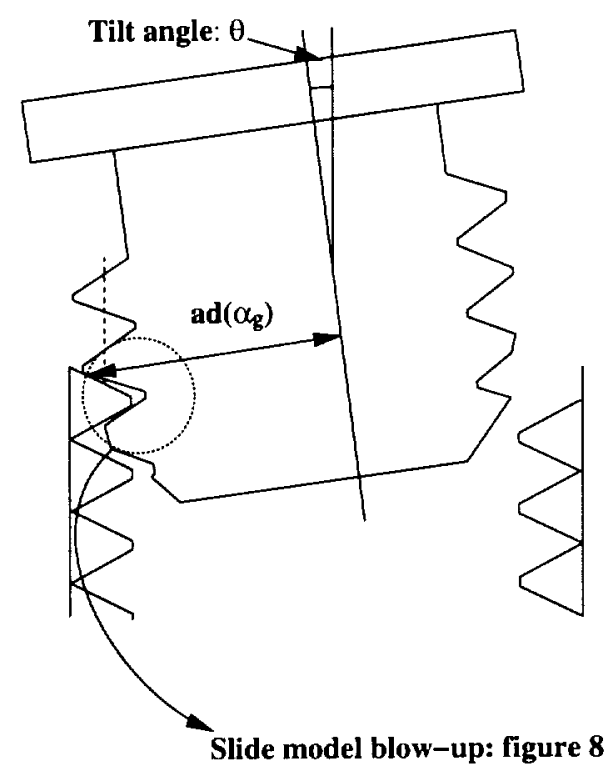

Figure 7: Slide model

$$
\begin{gathered}
h s=D N C * 2+d 3 * \cos 60, \\
c s=d 3 * \sin 60 .
\end{gathered}
$$

where $c s$ and $h s$ yield the relative position of the bolt thread with respect to the nut thread.

Combining the data for both the two point contact model and the slide model yields a plot of displacement change along the bolt's vertical axis: figure 9. As illustrated in the figures the slide model represents smaller drops than the two point model. In addition the discontinuity seen in the drop model signifies the switch from the aligned to the cross threading condition discussed above. The two models bound the possible displacement as a function of global tilt angle, $\theta$. If the effect of thread interaction outside of the global plane is minimal then the two point model will dominate as the bolt comes to rest in the nut after a drop. If out of plane thread interaction is significant, then the displacement will 


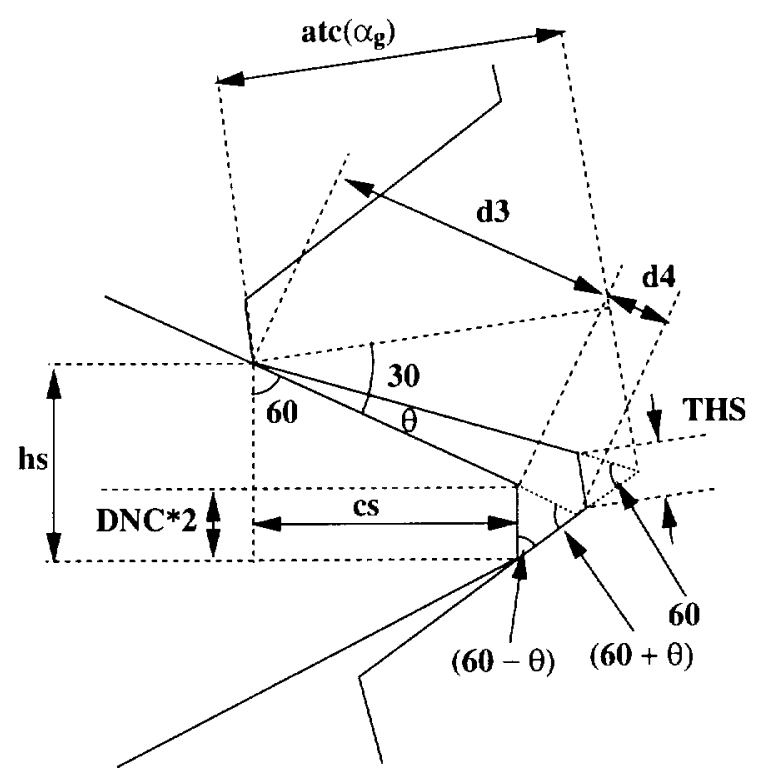

Figure 8: Slide model blow-up

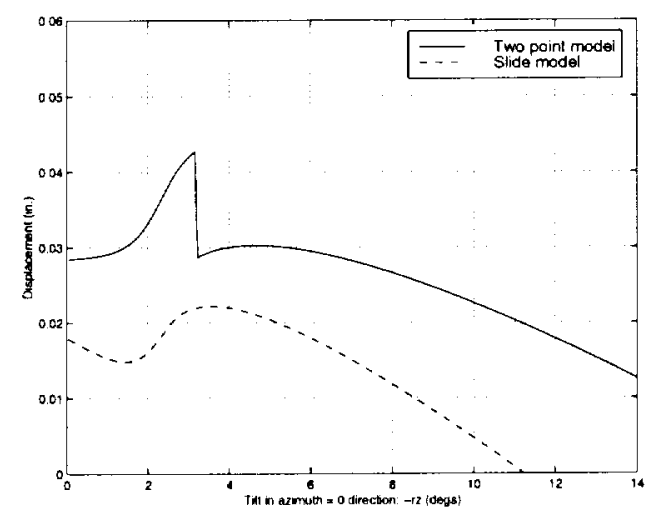

Figure 9: Theoretical drop data

rest between the two plots.

Both drop models show very similar displacements for significantly different tilt magnitudes. For example, the two point model shows approximately the same displacement for both a 0 degree tilt and a 7 degree tilt (figure 9). Additional information is needed to distinguish between these two cases. As will be seen in later sections covering experiments with actual hardware, this additional information is found in the displacement time history over a complete backspinning of one threaded part with respect to another. 
The models for the drop azimuth and the drop distance predict two important parameters associated with backspinning threaded parts. In the next section an instrumented fixture is used to validate these models and gain further insight into thread kinematics and dynamics.

\section{Experimental Setup}

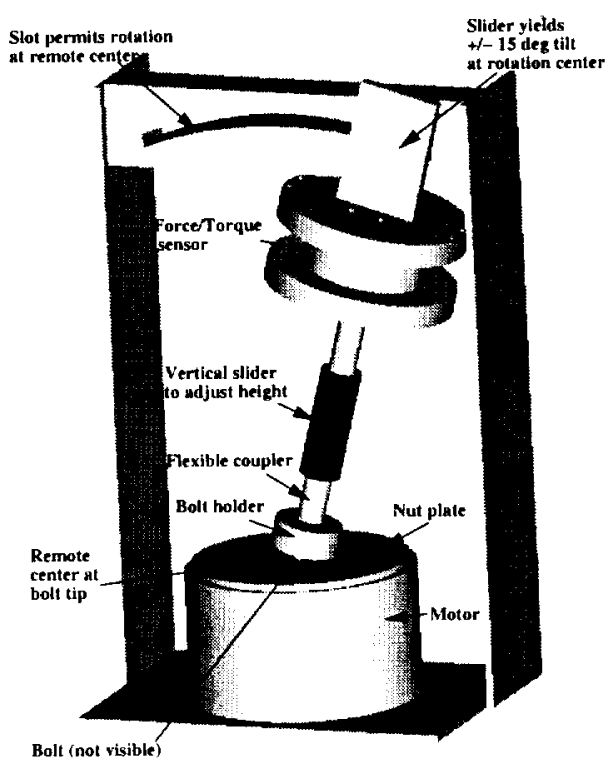

Figure 10: Fixture

The fixture shown in figure 10 is designed to provide high resolution force data for correlation with the models outlined in the previous section. The intent is to obtain additional data beyond what was reported in [4] using the Stanford/JPL hand, but the fixture is still only a tool which will help with the overall goal of implementing an alignment verification and correction scheme using the hand.

The fixture's translational degree of freedom permits the coupler to be compressed to apply a range of preloads from 0 to approx. $9 \mathrm{lbs}$. The energy stored in the coupler takes the place of the electronic compliance used in the hand. As the nut spins the bolt moves along 
its vertical axis, slightly releasing and then compressing the coupler axially. The resulting displacements are seen as force changes at the force/torque sensor which is nominally aligned with the bolt.

The compliance in the coupler will allow slight misalignments of approx. 1 degree). The axial force change, $\Delta f$ is directly proportional to the change in bolt axial displacement $\Delta x$ by:

$$
\Delta f=k_{\text {coupler }} \Delta x
$$

where $k_{\text {coupler }}$ is the coupler's axial stiffness, which is measured to be $26 \mathrm{lb}$./inch in the 4 to $9 \mathrm{lb}$. range. The alignment between the force/torque sensor and the bolt is far more precise than that which may be achieved with the hand.

The fastener set consists of a $1 / 2-13 \mathrm{UNC}-2 \mathrm{~A}$ (.5" diameter) steel bolt held beneath the coupler and a nut plate with mating steel $H E L I C O I L^{T M}$ threads mounted to a direct drive motor (figure 10). This setup, while not appropriate for practical applications, provides well controlled rotary motion between the fasteners and is extremely useful in isolating the change in the azimuth position when the bolt "drops" into the nut. It also parallels the setup with the Stanford/JPL hand which is described in the implementation section (section V).

Force time histories are collected as the nut backspins with respect to the bolt. Data is collected for all axes using a 6 axis force/torque sensor, but the only useful information for correlation with the drop models is found in the force that runs along the axis of the bolt. The force change along the bolt is correlated to the predicted changes in distance along the bolt's axis that occur when the bolt falls into the nut. 


\section{Results}

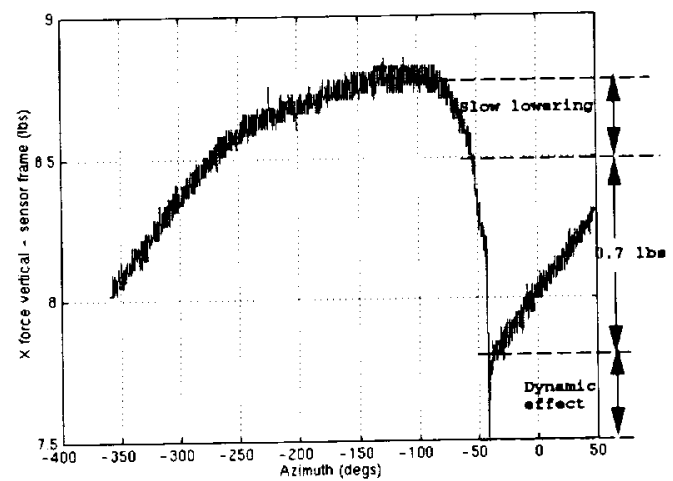

Figure 11: Tilt angle $=0 \operatorname{degs}-r_{z}$ direction

Figure 11 shows the distinct signature that occurs as the bolt drops into the nut for an approximate 0 degree tilt in the $-r_{z}$ direction. This figure shows force vs nut azimuth. This is essentially a time history of the force on the bolt as the nut spins but is plotted against azimuth to permit easy identification of the drop azimuth, $\alpha$. Azimuth refers to the motion of the nut thread start with respect to the bolt thread start and is defined the same way as drop azimuth, $\alpha$, in figure 1 . Specifically, drop azimuth, $\alpha$, is a parameter used to determine tilt angles for a specific drop, while azimuth is the more general position indicator. The azimuth history given in figure 11 reveals a slow lowering of the bolt into the nut just before the drop occurs. The lowering is a result of the bolt thread moving down along the nut thread start until it reaches the drop point.

The models presented in the thread kinematics section are quasistatic by design and only yield information applicable to the steady state force change that occurs over a drop. The undershoot in the force seen as the spike (figure 11) that terminates at approximately $7.5 \mathrm{lbs}$ is a dynamic/impact effect. The steady state force change must be measured with respect to 
the more slowly varying force levels that occur immediately after the spike. The steady state force change that is representative of the modeled drop is approximated as $0.7 \mathrm{lbs}$ (Figure 11). The nut is rotating at approximately 20 degrees/second which translates into a total time for this drop to be less that .5 seconds.

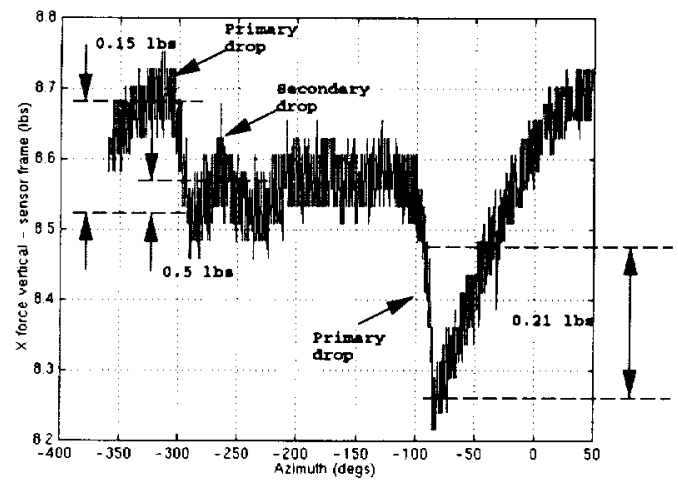

Figure 12: Tilt angle $=10 \mathrm{degs}-r_{z}$ direction

At moderate to high tilt angles, force time histories become more complicated and it becomes necessary to distinguish between different types of force changes. Figure 12 gives the time history for a 10 degree tilt in the same direction. In this case, there are two relatively rapid force changes and one significantly slower one. The rapid force changes are indicative of a drop as modeled in this paper and are defined to be primary drops. The slower change is not indicative of a primary drop and it is clear that the models developed in this work may not apply in explaining this drop. Given the ambiguity associated with this force change, it and other slow force changes like it are labeled secondary drops.

\subsection{Drop Azimuth Correlation}

Figure 13 shows the correlation in the $-r z$ direction, i.e. a tilt around the $\mathrm{z}$ axis in the negative direction (figure 1). Overall correlation is acceptable and is even better with only 
primary drops considered. However there is one set of 3 drops that are not predicted well. Once again, the vertical line at 90 degrees in figure 13 represents a sign change and is not part of the theoretical model. It is a graphical artifact associated with $\cos 90$.

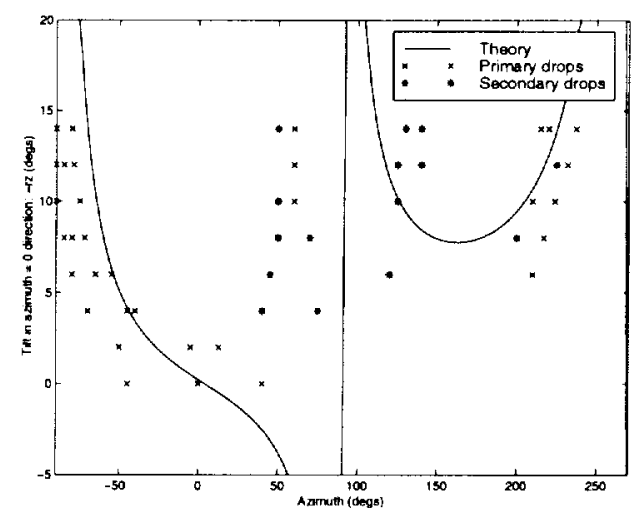

Figure 13: Tilt angle vs. drop azimuth $\left(-r_{z}\right.$ direction

Data in the $+r z-r y$ directions are similar to the $-r z$ direction and are all acceptable once small parameter adjustments are made for bolt and nut tolerances [1]. However, the data in the $+r_{y}$ direction is significantly different from the other 3 directions. Figure 14 shows the comparison between the test data and the predicted drops in the $+r_{y}$ direction. All of the data for tilt angles, $\theta$, above 4 degrees are secondary drops.

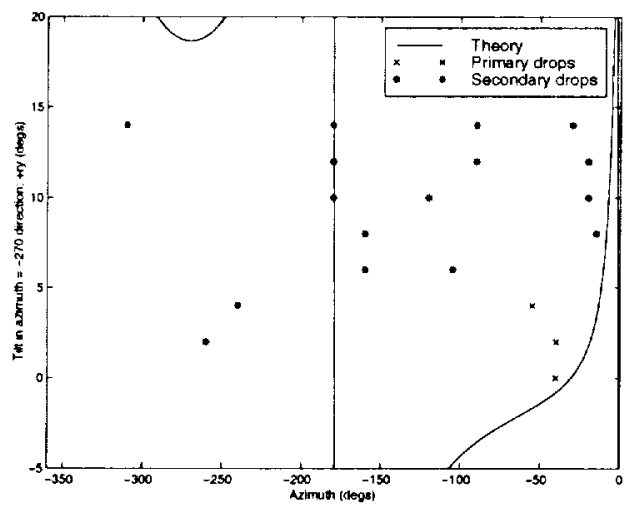

Figure 14: Tilt angle vs. drop azimuth $\left(r_{y}\right.$ direction)

As noted above, the secondary drops are not modeled by the kinematic mechanisms 
developed in this work. It is actually not clear what physical mechanism yields the slowly varying force time histories. But it is likely that the mechanism(s) are related to drop models outlined in this paper. As their very names state, both the primary and secondary drops are associated with force minima. This likely relationship is borne out in the tendency for the higher tilt angle secondary drops to cluster in drop azimuth regions that are nearly perpendicular to the tilt direction. For figure 14 these azimuth regions are at 0 and -180 degrees.

\subsection{Force Drop Correlation}

Figure 15 gives the correlation between test data and the drop models for tilts in the $-r_{z}$ direction. Correlation is good up to approximately 10 degrees of tilt, after which the two point model indicates that the bolt no longer drops. The slope correlation, especially up to 10 degrees of tilt, is acceptable. Overall the data tends to fall closer to the two point model indicating that for most of the tilt range the bolt can fall the maximum distance predicted.

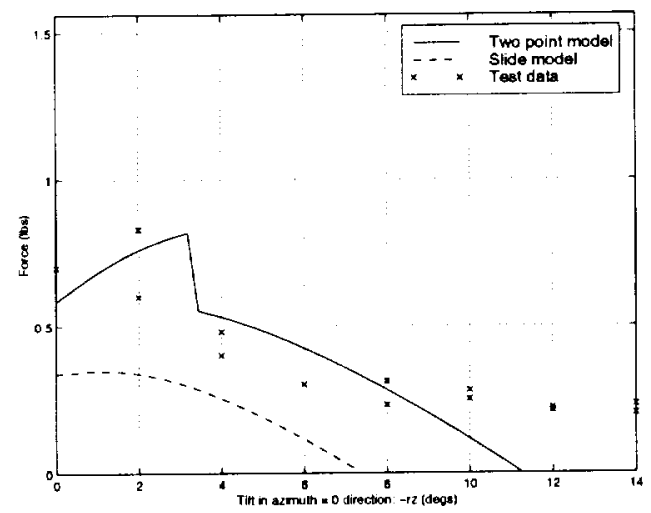

Figure 15: Force drop vs. tilt angle $-r_{z}$ direction.

The slope for the two point model is positive prior to cross threading. As seen in figure 15 this indicates that the largest force drop occurs at a tilt angle slightly before the 
cross threading tilt angle. (The discontinuity in the two point model, figure 15, signals the change from the non-cross threading mechanism to the cross threading one.) The test data supports the quasistatic/kinematic prediction. This important result (also seen in other tilt directions) indicates that the largest force drop, in general, does not indicate the best bolt/nut alignment. This is a counter intuitive result. Fortunately, the largest force drop is still associated with a non-cross threading condition and indicates that mating will be successful.

Similar force drop data is seen for the $-r_{y}$, and $+r_{z}$ direction, however no significant results can be presented for the $+r_{y}$ direction since the drop models do not predict the change in height due to the slowly varying secondary drops.

\subsection{Discussion}

Several important trends indicated by the theoretical model are borne out in the test data. The most important is that the drop azimuths (for the primary drops) approach directions nearly perpendicular to the tilt direction as the tilt angle increases. This effect is the best starting point for determining which way to tilt to correct alignment. In addition, the somewhat counter intuitive result that the largest force drop is not associated with perfect alignment is also confirmed by the test data. However, both test data and the two point drop model agree that the largest force drop still indicates that the bolt is sufficiently aligned to prevent cross threading. This is another result relevant to implementation since the largest force drop will clearly be used as an alignment indicator.

The data has also revealed phenomena not directly modeled by the kinematic mechanisms 
developed in this work. The most important unmodeled phenomena are the secondary drops that are indicative of out of plane thread interaction. It is not clear what causes these slower drops but they do share one important characteristic with the primary drops. The minima that occur after the secondary drops approach directions that are nearly perpendicular to the tilt direction as the tilt angle increases. This unifying characteristic between the secondary and primary drops gives support for using this perpendicular effect as the starting point for implementation techniques discussed next.

\section{Implementation}

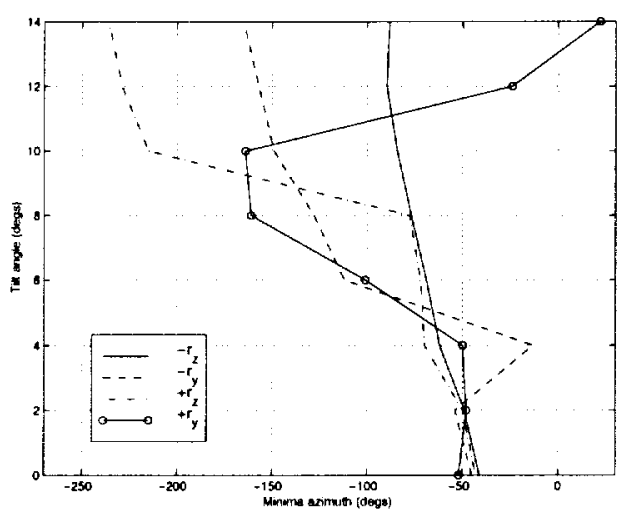

Figure 16: Tilt angle vs. minima azimuth - all directions

The overall goal for this work is to correct alignment between threaded parts with the Stanford/JPL hand. The primary drops which are modeled and validated in this work do not occur in all tilt directions in the tilt range of interest, and can therefore not serve as the sole source of information for correcting alignment.

An alignment technique is needed that encompasses all possible tilt directions. A good datum for use in developing such a comprehensive technique is the force minimum that 
occurs while backspinning. The azimuth associated with the force minimum is called the minima azimuth. Figure 16 shows the tilt angle vs minima azimuth for the four directions discussed in the previous section. Data in all directions correlate very well with the primary drops. Interestingly, the tilt angles in the $+r_{y}$ direction ( figure 14) also correlate with the secondary drops in a currently unpredictable way. The important perpendicular trend that occurs at the higher tilt angles is still present and provides, to date, the only consistent piece of information useful for determining the tilt direction and correcting misalignment using the JPL/Stanford hand.

\subsection{JPL/Stanford Hand}

The Johnson Space Center's Dexterous Anthropomorphic Robotic Testbed (DART) [6] is equipped with two JPL/Stanford hands [12]. These three-fingered-hands (figure 17) employ motor driven cable tendons to actuate the three joints on each finger. The tendons are routed through conduits to permit easy mounting of the hand at a distance from the motor assembly. The basic control system for the hands uses a layered impedance strategy [12], [17], [2]. Impedance loops control tendon tension, joint moments, Cartesian finger forces, and grasped object forces and moments. At the lowest level tendon tensions are maintained by driving each motor in velocity mode. The tension levels are read at strain gauges located directly behind the finger assemblies (figure 17) ensuring accurate tension control at a distance from the driving motors. 


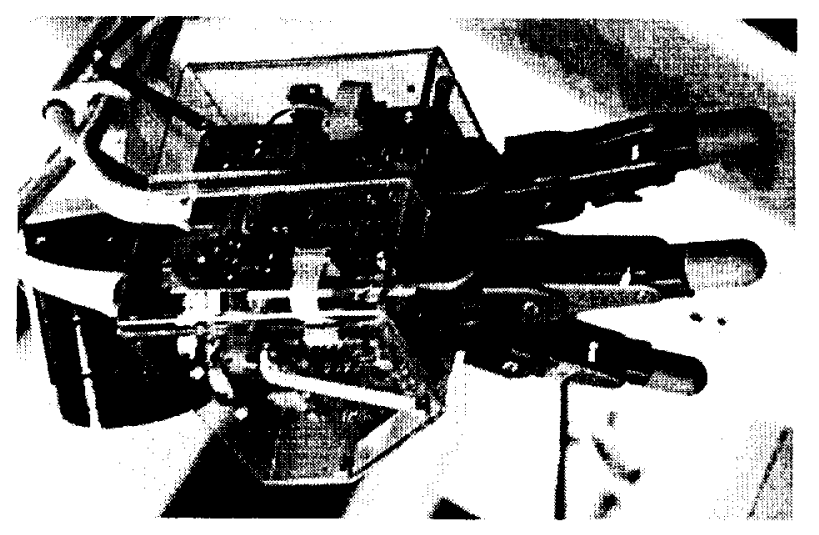

Figure 17: Salisbury Hand photograph

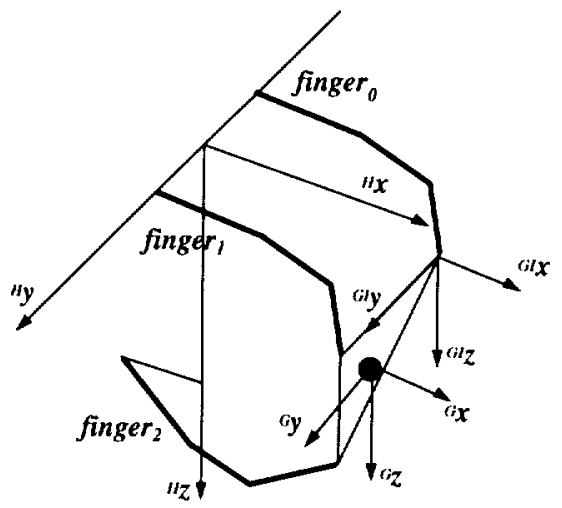

Figure 18: Hand Grasp Frame 


\subsection{Object Compliance Control}

For thread alignment experiments, the JPL/Stanford hand is configured in object grasp control mode, where the three fingers come together to grasp a bolt. In this configuration, the hand's 9 degrees of freedom (DoF) combine together to actuate 6 degrees of freedom rigid body object control at the grasp center which is located at the centroid of the triangle defined by the finger tips (figure 18). The three remaining degrees of freedom are used to maintain a positive force between each pair of fingers to prevent slip. Object force control is maintained by commanding the fingers in concert to yield forces and moments at the grasp center. The actual object force command, $\underline{O}$, is generated from position errors in the grasp frame to yield a stiffness control of the form:

$$
\underline{O}=[K] \underline{e},
$$

where the total force vector $\underline{Q}$ is given by:

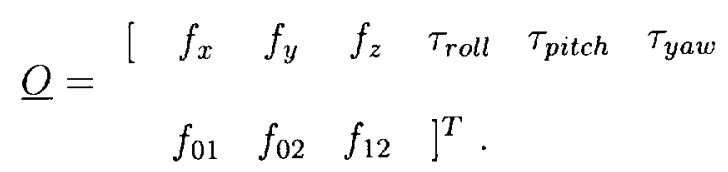

$[K]$ is a $9 \times 9$ diagonal matrix and its elements can be set to obtain an arbitrary stiffness in each axis, and

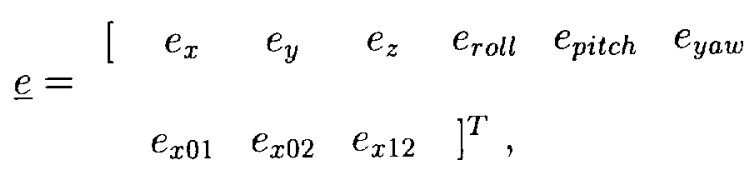

The rigid body elements of the object force, $\underline{Q}$ and the object position error, $\underline{e}$ have their 
standard meanings (fixed angles). The force element, $f_{01}$ refers to the force between fingers 0 and 1 along a vector between the tips, and $f_{02}$ and $f_{12}$ follow the same convention. The position error element $e_{x 01}$ is the error in the distance between finger tips 0 and 1 .

\subsection{Results}

To evaluate the Stanford/JPL hand's ability to correct alignment using the perpendicular effect, the hand is configured to hold a bolt at an angle between 10 and 14 degrees with respect to the rotating nut plate (figure 19). For this implementation, a new bolt not previously used in testing is used to evaluate the general nature of the results.

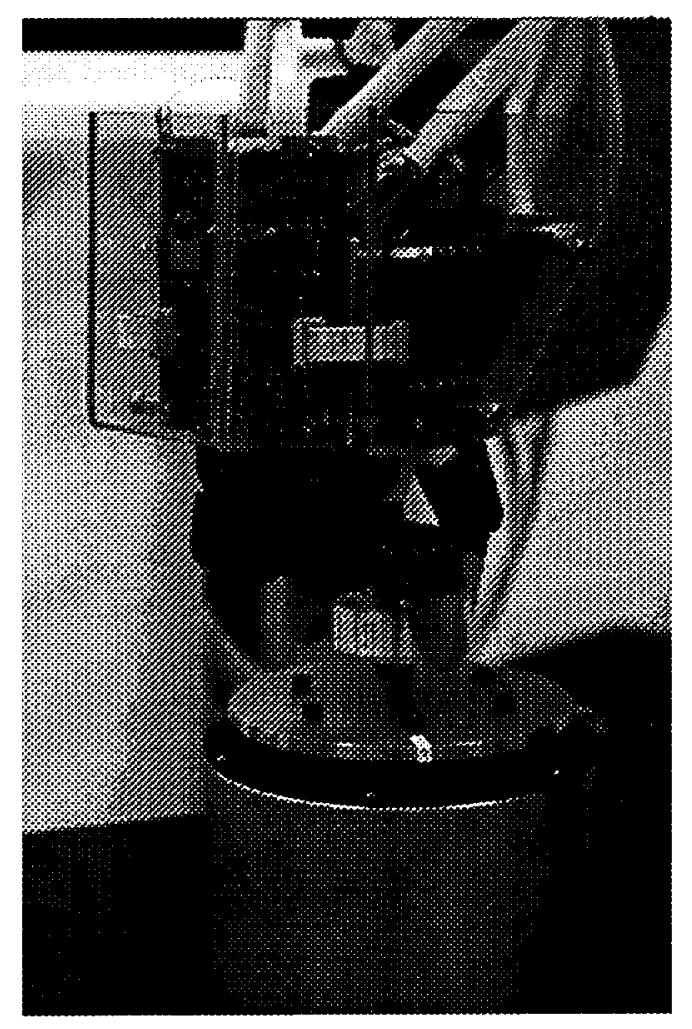

Figure 19: Implementation setup

As the nut plate rotates, the hand data collection routine searches for the minimum force value in real-time. The time corresponding to the minimum is transferred to the 
program controlling the motor that drives the nut plate. This routine calculates the azimuth corresponding to the minimum and then increases it by 90 degrees to yield the predicted tilt direction. The motor then drives the nut thread start to the predicted tilt direction for use as a visual cue during testing.

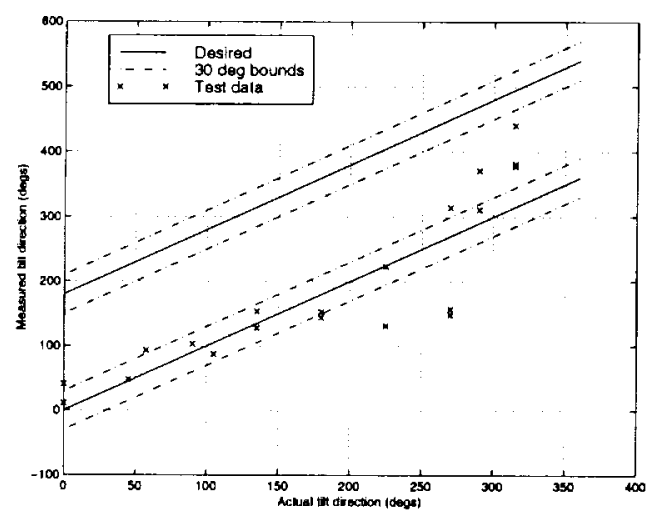

Figure 20: Hand predictions for tilt direction

Twenty tests were performed and the resulting data is given in figure 20 . The tilt direction as predicted by the hand is plotted against the actual tilt direction. For 9 out of 20 cases, the predicted data fell within 30 degrees of the actual tilt direction. The two desired lines shown in this figure refer to the actual tilt direction and the negative (or 180 degree out of phase) tilt direction. The negative direction is included since the perpendicular effect does not specify whether the difference between the minima azimuth is positive or negative 90 degrees. Overall 9 out of 20 cases correspond to a mediocre result and is indicative of the hand's hardware limitations, specifically, the lack of position sensors at the joints [1].

After determining the tilt direction and driving the nut thread start to the azimuth corresponding to the predicted tilt direction, the system commands the hand to correct alignment by tilting $10-14$ degrees in the predicted direction. This large range is required since the hand's lack of joint sensors make accurate predictions of the actual tilt change 
unreliable. The hand software does not try to verify alignment since its force sensing system limits alignment predictions to 5 degrees [1]. Out of the 9 good predictions, noted above, the hand is able to correct alignment ( as measured visually) 4 times. Considering the hands limitations, this is actually better than expected.

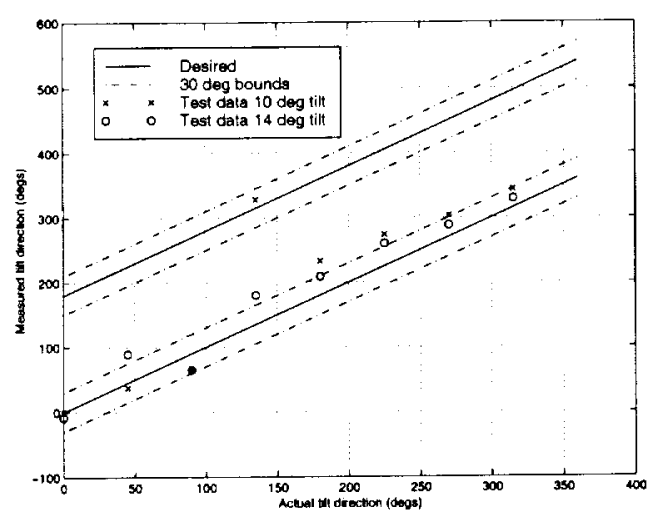

Figure 21: Fixture predictions for tilt direction

The same bolt used in the alignment correction evaluation with the hand is tested in the fixture. As expected the higher resolution fixture yields significantly better results. For 10 out of 16 tests, predicted tilt directions fall within 30 degrees of the actual direction, and all the rest fall within approximately 60 degrees. The fixture can only tilt in one plane and is not motorized and therefore can not be used to correct alignment.

The 30 degree boundaries are used as measures of the quality of the predicted answer. An alignment correction within 30 degrees ensures that $86 \%$ of the adjustment is in the correct direction while only $50 \%$ is in the incorrect direction.

The proposed strategy for using this perpendicular effect is to first determine which direction to tilt in: 90 degrees past the minima azimuth or 90 degrees before the minima azimuth. This can be accomplished by making two relatively large tilts in either direction and determining which one yields the larger force drops. The first choice is +90 degrees 
which corresponds to the desired line running through 0 degrees in figure 21 . After the $+90,-90$ choice is made, the system tilts 10 to 12 degrees in the predicted tilt direction, monitors the force drop, then goes back to the attitude before the tilt, and chooses another direction 15 to 30 degrees off from the original, until alignment is indicated. Basically, the implementation is designed to narrow the range of tilt direction choices from 0 through 360 degrees, to 60 through 90 degrees around a predicted direction. The result is expected to be a reduction of between $75 \%$ to $83 \%$ in the region of tilt directions that must be evaluated to determine the proper direction to correct alignment.

\section{Conclusions}

This paper has examined the possibility of using information gained by backspinning a bolt into a nut to improve the automation and robotic mating of threaded parts. A new model for the kinematics and statics of backspinning had been presented, and empirically verified via the use of a specialized fixture. Based on this model, a new strategy for determining and correcting alignment has been implemented with the Stanford/JPL robotic hand.

The judicious use of backspinning appears to present a number of significant advantages for threaded robotic and automated assembly. It is clear that multiple force changes occur while backspinning a threaded insert nut with respect to a moderately misaligned bolt. The multiple force drops yield a clear indication of misalignment. This signature can be used to augment or replace previous torque signature techniques that only provide information after cross threading has occurred and parts have been damaged.

The kinematic/quasistatic models presented in this paper provide insight into the in- 
teraction that occurs between threaded parts when they are backspinning with respect to each other. The general tendency for the tilt direction to be approximately 90 degrees out of phase with the drop azimuth for large tilt misalignments is one of the most important results. In addition this 90 degree rule is extendable to a class of force drops (secondary drops) that are not directly related to the kinematic models developed in this work. Based on this trend a technique has been developed, and tested to the degree possible with the Stanford/JPL hand, that should at least moderately improve current alignment techniques.

\subsection{Future Work}

Since the tilt angle vs minima azimuth data (figure 16) do not exhibit a linear trend, one future implementation strategy would be to build a complex map using test data with several bolts. A software routine would use this map along with force and relative azimuth data collected during backspinning at one or more unknown tilt angles to predict the direction and magnitude of the misalignment.

\section{Acknowledgments}

The authors would like to thank Larry Li, Chris Lovchik, Charles Price, and Edith Taylor of the NASA/Johnson Space Center, and Michael Ross and West Womack of Lockheed Martin for their encouragement and continuing support throughout this work. Funding is partially provided by NASA JSC under grant NAG 9-845, the National Science Foundation under grant CMS 9532081 and the office of Naval Research under contract N00014-96-C-0320. 


\section{References}

[1] M. A. Diftler. Alignment of Threaded Parts Using a Robot Hand: Theory and Experiments. PhD thesis, Rice University, Houston, TX, 1997.

[2] M. A. Diftler and I. D. Walker. Compliance Control for the Dexterous Anthropomorphic Robotic Testbed (DART) Stanford/JPL Hands. Technical Report TR95-17. Technical report, Rice University, Electrical and Computer Engineering, 1995.

[3] M. A. Diftler and I. D. Walker. Experiments in mating threaded parts using a dexterous robotic hand. In Proc. Symposium on Robotics and Cybernetics, CESA'96 IMACS Multiconference, pages 459-464, 1996.

[4] M. A. Diftler and I. D. Walker. Determining alignment between threaded parts using force and position data from a robot hand. In Proc. IEEE Int. Conf. Robotics Automat., pages $1503-1510,1997$.

[5] A. Kato. Valuation of the practical use of SCARA type robots on assembly lines and the future robotized assembly systems. In Int. Symp. on Robotics Research, pages 479-486, 1985.

[6] L. Li, B. Cox, S. Shelton, and M. Diftler. Telepresence Control of an Ambidextrous Robot for Space Applications. Report JSC-33306. Technical report, NASA Johnson Space Center, 1995. 
[7] M. Matsumura, S. Itou, H.Hibi, and M. Hattori. Tightening torque estimation of a screw tightening robot. In Proc. IEEE Int. Conf. Robotics Automat., pages 2108-2112, 1995.

[8] P. Michelman and P. Allen. Forming complex dexterous manipulations from task primitives. In Proc. IEEE Int. Conf. Robotics Automat., pages 3383-3388, 1994.

[9] E. J. Nicolson and R. S. Fearing. Compliant control of threaded fastener insertion. In Proc. IEEE Int. Conf. Robotics Automat., pages 484-490, 1993.

[10] S. N. Osipov and A.M. Formal'skii. On the theory of manipulation systems with force sensitization. Mechanics of Solids, 22(2):62-70, 1987.

[11] Panasonic. Panasonic Screw Fastening Robot. Technical report, Panasonic Factory Automation, 9377 West Grand Ave., Franklin Park, IL.

[12] J. K. Salisbury and M. T. Mason. Robot Hands and the Mechanics of Manipulation. MIT Press, Cambridge, MA, 1985.

[13] H. Sato and et. al. Development of a model based remote maintenance robot system (I) - outline of the robot system. In Proc. IEEE Int. Conf. Intell. Robots and Systems, pages $1225-1230,1993$.

[14] B. Smith. Automatic system for accurate fastening. Machine Design, 61(17):44-48, 1989.

[15] S. K. Smith. Use of a microprocessor in the control and monitoring of air tools while tightening threaded fasteners. In Proc. SME Autofact West, pages 387-421, 1980. 
[16] T. Speeter. Primitive based control of the Utah/MIT dexterous hand. In Proc. IEEE Int. Conf. Robotics Automat., pages 866-877, 1991.

[17] G. P. Starr. Experiments in assembly using a dexterous hand. IEEE Trans. Automat. and Robotics, 6(3):342-347, 1990.

[18] J. M. Tao, J. Y. S. Luh, and Y. F. Zheng. Compliant coordination control of two moving industrial robots. IEEE Trans. Automat. and Robotics, 6(3):322-330, 1990.

[19] T. Tsujimura and T. Yabuta. Adaptive force control of screw driving with a positioning controlled manipulator. Robotics and Autonomous Systems, 7(1):57-65, 1991. 